$02 ; 12 ; 13$

\title{
Многоканальный энергоанализатор заряженных частиц из двух коаксиальных цилиндров с дискретным внешним цилиндром и плоскими торцевыми электродами
}

\author{
() Т.Я. Фишкова
}

Физико-технический институт им. А.Ф. Иофффе РАН, Санкт-Петербург E-mail: fishkova@mail.ru

Поступило в Редакцию 22 декабря 2016 г.

Путем компьютерного моделирования найдены параметры предложенного автором светосильного многоканального анализатора заряженных частиц по энергии простой конструкции в виде цилиндрического конденсатора с дискретным внешним цилиндром и закрытыми торцами в широком диапазоне одновременно регистрируемых энергий $\left(E_{\max } / E_{\min }=100\right)$. При введении дополнительного цилиндрического электрода малых размеров вблизи переднего торца системы удается более чем на порядок улучшить разрешение в области малых энергий. При этом разрешение энергоанализатора во всем указанном диапазоне энергий составляет $\rho=(4-6) \cdot 10^{-3}$.

DOI: 10.21883/PJTF.2017.11.44693.16653

Для обеспечения экспресс-анализа состава материалов, технического контроля изделий различных отраслей промышленности, а также ввиду широких исследований различного рода биологических объектов, в частности радиационной терапии, по энергетическому спектру вторичных частиц, исходящих с поверхности или из объема тех или иных веществ, используются многоканальные электростатические анализаторы (спектрографы). Основная проблема состоит в создании распределения электростатического поля, позволяющего одновременно фокусировать электронные потоки с энергиями, различающимися в десятки раз, причем объект исследования и детектор должны быть вынесены за пределы поля. Поставленная проблема решается предло- 
женным автором светосильным спектрографом [1], рассмотренным и усовершенствованным в настоящей работе.

Ранее был исследован спектрограф [2], представляющий собой цилиндрический конденсатор с разрезанным внешним цилиндром, на части которого подаются напряжения, возрастающие по линейному закону. Однако диапазон одновременно регистрируемых энергий $E$ у него не превышает величины $E_{\max } / E_{\min }=24$, причем лишь в случае пренебрежения краевыми эффектами, возникающими из-за наличия у системы открытых торцов. Следует отметить также, что в спектрографе [2] исследуемый объект - источник вторичных электронов, а также источники различного рода первичных излучений могут располагаться лишь внутри цилиндрического электрода меньшего диаметра, что непригодно для практического использования.

Интерес представляют спектрографы с плоскими электродами, свободные от указанных выше конструктивных недостатков, в которых удается расширить диапазон одновременного энергоанализа сфокусированных на плоский позиционно-чувствительный детектор пучков заряженных частиц до $E_{\max } / E_{\min }=50$ при вынесенных за пределы поля исследуемом объекте и детекторе [3]. Однако существенным недостатком спектрографов из плоских электродов является малая светосила, поскольку фокусировка пучков заряженных частиц может быть осуществлена лишь в одной плоскости.

Задача о нахождении электронно-оптических свойств предложенного светосильного спектрографа [1], а также его усовершенствованной конструкции решается по написанной с участием автора настоящей работы программе ТЕО для расчета различного рода полей и траекторий заряженных частиц в осесимметричных и плоских электростатических системах. Результаты проверки точности расчетов по ТЕО плоского и цилиндрического конденсаторов, для которых имеются аналитические выражения для полей, а также аналитические решения дифференциальных уравнений траекторий заряженных частиц, показали, что расхождения не превышают $(0.1-0.5) \%$ в зависимости от размеров ячеек сетки в программе ТЕО. Исследованная в настоящей работе конструкция спектрографа схематически представлена на рис. 1 . Он состоит из коаксиально расположенных внешнего и внутреннего цилиндрических электродов, а также плоских электродов, закрепленных на торцах внутреннего цилиндра, которые не только участвуют в образовании требуемого для решения задачи электростатического поля, но

Письма в ЖТФ, 2017, том 43, вып. 11 


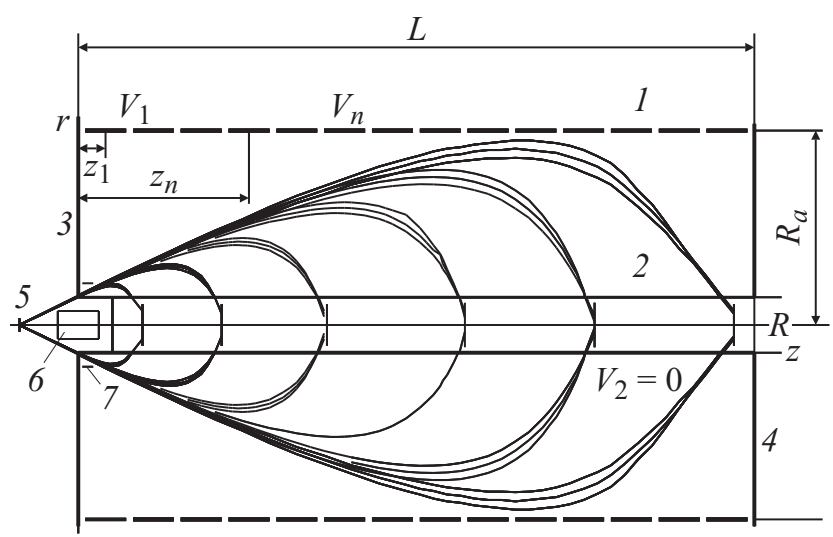

Рис. 1. Схема светосильного спектрографа с дополнительным цилиндрическим электродом и траектории пучков заряженных частиц при отношениях энергий пучков к их минимальной энергии, последовательно равных $E / E_{\min }=1,10,25$, $50,75,100.1-$ внешний разрезной цилиндрический электрод, $2-$ внутренний цилиндрический электрод, 3,4 - плоские электроды по торцам системы, 5 исследуемый объект, 6 - источник первичных электронов либо другого рода излучений, 7 - дополнительный цилиндрический электрод.

и одновременно препятствуют проникновению в рабочее пространство посторонних полей.

Внешний цилиндрический электрод выполнен из $n \geqslant 10$ цилиндрических электрически изолированных частей одинакового радиуса, подсоединенных к источнику питания, выполненному с возможностью подачи на части внешнего электрода линейно возрастающего напряжения. Внутренний цилиндрический электрод заземлен и снабжен выходными кольцевыми щелями, затянутыми металлической сеткой.

Длина цилиндрических частей внешнего электрода удовлетворяет соотношению $\ln \leqslant 2 R$, расстояние между ними удовлетворяет соотношению $s_{n} \leqslant 0.5 R$, где $R$ - радиус внутреннего цилиндрического электрода. Следует отметить, что здесь и в дальнейшем все линейные размеры, а также геометрические параметры спектрографа: положение объекта и точек фокусировки пучков различных энергий, коэффициенты линейной дисперсии по энергии - выражены в единицах $R$. Поэтому, выбрав любой нужный для решения той или иной задачи

Письма в ЖТФ, 2017, том 43, вып. 11 
размер внутреннего цилиндрического электрода, можно определить все геометрические и электронно-оптические параметры предлагаемого устройства.

Исследуемый объект (источник вторичных электронов), а также источник первичных электронов (электронная пушка) либо источник другого рода излучений размещены за пределами поля. Детектор состоит из набора плоских электродов, которые подключены к многоканальному измерительному устройству и установлены за выходными щелями во внутреннем цилиндре перпендикулярно его оси.

После необходимой оптимизации путем численных расчетов по программе ТЕО выбраны геометрические параметры исследуемого в настоящей статье спектрографа. Они таковы: внешний цилиндрический электрод имеет радиус $R_{a}=7 R$ и выполнен из $n=14$ электрически изолированных частей одинакового радиуса, длина которых равна $l_{n}=1.5 R$ при расстоянии между ними, равном $s_{n}=0.25 R$; продольный размер системы - расстояние между торцевыми электродами менее чем в два раза превышает поперечный и равен $L=24.5 R$. Части разрезного внешнего цилиндра подсоединены к источнику питания, обеспечивающему возможность подачи на них напряжения, возрастающего по линейному закону. При этом величины потенциалов на частях дискретного электрода равны $V_{n}=V_{1}\left(z_{n} / z_{1}\right)$, где $V_{1}-$ потенциал на первой части дискретного электрода, $z_{n}=z_{1}[1+1.75(n-1)]-$ расстояние от середины каждой части до переднего торца системы, $n-$ порядковый номер соответствующей части.

Как показали расчеты автора, приведенные в заявке на полезную модель [1], для описанной системы диапазон одновременно регистрируемых энергий при фокусировке пучков различных энергий на внутренний цилиндрический электрод достигает $E_{\max } / E_{\min }=50$. В настоящей работе исследовалась возможность увеличения диапазона энергий за счет изменения трех независимых параметров: питающих потенциалов на разрезном цилиндрическом электроде; положения источника $z_{i}$ (расстояние вдоль продольной оси системы от объекта до передней стенки спектрографа), а также углов впуска пучков заряженных частиц в поле. Компьютерное моделирование описанного выше спектрографа показало, что оптимальными оказались $V_{1}=\left(4.5 E_{\min }\right) / e$, расстояние от переднего торца системы до исследуемого объекта, вынесенного за пределы поля, $z_{i}=2.2 R$ и начальные углы впуска пучков заряженных частиц различных энергий равны $\theta=25.5^{\circ} \pm 0.5^{\circ}$. При указанных

Письма в ЖТФ, 2017, том 43, вып. 11 


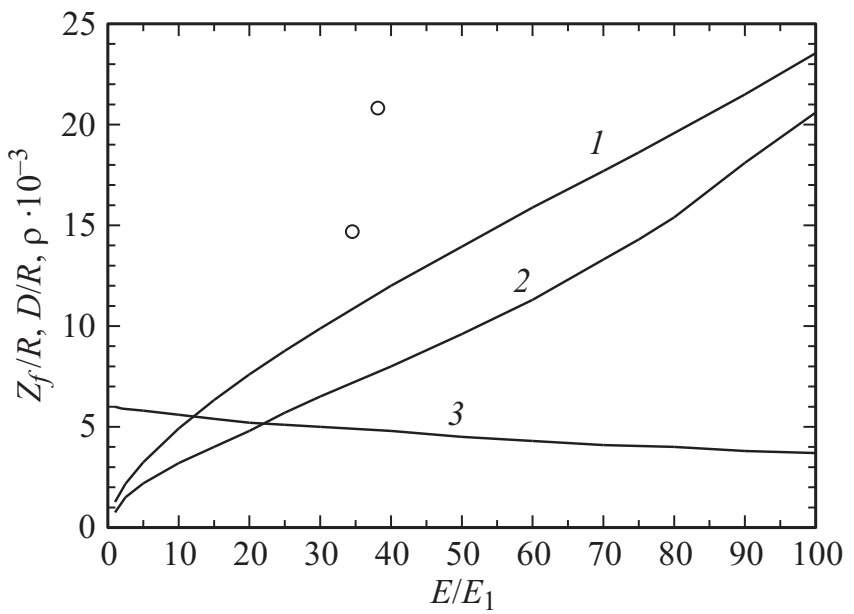

Pис. 2. Основные параметры цилиндрического спектрографа в широком диапазоне регистрируемых энергий $E_{\max } / E_{\min }=100: 1-$ положение точек фокусировки на внутреннем цилиндре $z_{f} / R, 2-$ величина коэффициента линейной дисперсии по энергии $D / R, 3$ - энергетическое разрешение спектрографа $\rho$.

параметрах диапазон одновременно регистрируемых энергий может быть повышен до $E_{\max } / E_{\min }=100$. На рис. 2 приведены основные рабочие характеристики спектрографа в указанном диапазоне энергий: $z_{f} / R$ - положения центральных траекторий, сфокусированных на внутренний цилиндр кольцевых пучков, $D / R$ - величины коэффициентов линейной дисперсии по энергии, а также разрешение спектрографа по энергии $\rho=\Delta E / E$. Следует отметить, что хорошее разрешение для малых энергий в области $E / E_{\min }=(1-10)$ достигнуто благодаря введению вблизи области прохождения пучков рядом с передним торцом спектрографа дополнительного цилиндрического электрода малых размеров. Численные расчеты показали, что для него оптимальными являются следующие геометрические параметры: радиус $r=1.5 R$, длина $l=0.3 R$, расстояние от переднего торца системы $s=0.05 R$, потенциал дополнительного электрода оказался равным $u=0.1 V_{1}$. Такой электрод значительно улучшает фокусировку на внутренний цилиндр пучков малых энергий, что ведет к существенному улучшению разрешения

Письма в ЖТФ, 2017, том 43, вып. 11 


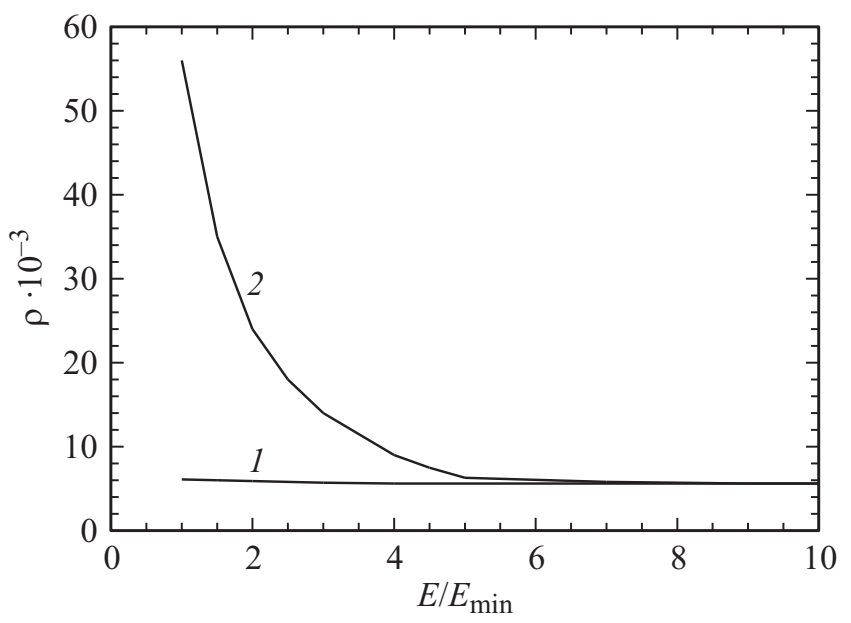

Рис. 3. Разрешение спектрографа для малых энергий: 1 - при наличии дополнительного электрода, 2 - в случае его отсутствия.

спектрографа по энергии (см. кривую 1, рис. 3). Для сравнения на этом рисунке дана кривая 2 , которая соответствует разрешению по энергии в отсутствие дополнительного электрода. Видно, что для минимальной энергии при использовании дополнительного электрода выигрыш в разрешении составляет более 50 раз. Для средних и больших энергий в диапазоне $10<E / E_{\min }<100$ наличие дополнительного малого цилиндрического электрода на фокусировку не влияет.

В заключение следует отметить, что предложенный автором и оптимизированный в настоящей статье электростатический спектрограф из двух коаксиальных цилиндров с разрезным внешним цилиндром и плоскими торцевыми электродами является лучшим из всех известных к настоящему времени спектрографов из-за большой светосилы, которая примерно на два порядка выше, чем у различных спектрографов, состоящих из плоских электродов, один из которых разрезан на части. При этом главные параметры, а именно диапазон одновременно регистрируемых энергий и разрешение по энергии предлагаемого спектрографа из цилиндрических электродов, не уступают лучшим системам из плоских электродов.

Письма в ЖТФ, 2017, том 43, вып. 11 


\section{Список литературы}

[1] Фишкова Т.Я. Электростатический анализатор энергии заряженных частиц: Решение от 30.11.2016 о выдаче патента на полезную модель № 2016122283 от 06.06.2016.

[2] Read F.H. // Rev. Sci. Instr. 2002. V. 73. N 3. P. 1129-1139.

[3] Фишкова Т.Я. // Прикладная физика. 2015. № 2. С. 101. 